\section{Mutual genetic antagonism involving GLI3 and dHAND prepatterns the vertebrate limb bud mesenchyme prior to SHH signaling}

\author{
Pascal te Welscher, ${ }^{1}$ Marian Fernandez-Teran, ${ }^{2}$ \\ Marian A. Ros, ${ }^{2}$ and Rolf Zeller ${ }^{1,3}$ \\ ${ }^{1}$ Department of Developmental Biology, Faculty of Biology, \\ Utrecht University, 3584CH Utrecht, The Netherlands; \\ ${ }^{2}$ Department of Anatomy and Cell Biology, Facultad de \\ Medicina, Universidad de Cantabria, 39011 Santander, Spain
}

The bHLH transcription factor dHAND is required for establishment of SHH signaling by the limb bud organizer in posterior mesenchyme, a step crucial to development of vertebrate paired appendages. We show that the transcriptional repressor GLI3 restricts $d H A N D$ expression to posterior mesenchyme prior to activation of SHH signaling in mouse limb buds. dHAND, in turn, excludes anterior genes such as Gli3 and Alx 4 from posterior mesenchyme. Furthermore, genetic interaction of GLI3 and dHAND directs establishment of the SHH/ FGF signaling feedback loop by restricting the BMP antagonist GREMLIN posteriorly. These interactions polarize the nascent limb bud mesenchyme prior to $\mathrm{SHH}$ signaling.

Received October 25, 2001; revised version accepted December 28, 2001.

Development of paired appendages (limbs and fins) in vertebrates is controlled by a mesenchymal organizer located at the posterior limb bud margin (Johnson and Tabin 1997). Analysis of chicken and mouse limb bud development has shown that Sonic Hedgehog (SHH) is the morphogenetic signal expressed by the organizer (called polarizing region, or ZPA) that controls patterning of the distal limb bud (Chiang et al. 2001; Kraus et al. 2001). During progression of limb bud development, the polarizing region is maintained and propagated distally by an SHH/FGF signaling feedback loop. The SHH/FGF feedback loop is established in the posterior mesenchyme through localized activation of the BMP antagonist GREMLIN, which relays the SHH signal from responding mesenchymal cells to FGFs expressed by the posterior apical ectodermal ridge (AER; Zuniga et al. 1999; Sun et al. 2000). In turn, FGF signaling by the posterior AER maintains the SHH signaling polarizing region (Johnson and Tabin 1997). Interestingly, both GREMLIN in the posterior mesenchyme and FGFs in the

[Key Words: Alx4; dHAND; Gli3; limb development; polarizing region; SHH signaling]

${ }^{3}$ Corresponding author.

E-MAIL R.Zeller@bio.uu.nl; FAX 31-30-254-2219.

Article and publication are at http://www.genesdev.org/cgi/doi/10.1101/ $\operatorname{gad} .219202$. posterior AER are activated prior to SHH signaling (Zuniga et al. 1999), similarly to other posterior genes such as $5^{\prime}$ HoxD genes (Kraus et al. 2001). These studies indicate that the nascent limb bud mesenchyme is already prepatterned (Chiang et al. 2001) and that the mesenchymal responsiveness to polarizing region signals is established prior to SHH activation. However, little is known about the mechanism acting upstream of SHH signaling to polarize the limb field and early limb bud. Several studies suggest a possible combinatorial involvement of Hox genes in positioning and polarizing the early limb field in response to retinoic acid (Lu et al. 1997). In particular, anterior ectopic expression of Hoxb8 in forelimb buds of transgenic mouse embryos results in establishment of an ectopic polarizing region and mirror image duplication of distal limb structures (Charité et al. 1994). However, neither targeted inactivation of the Hoxb8 gene (van den Akker et al. 1999) nor deletion of all Hox8 paralogs in the mouse alters limb morphogenesis (van den Akker et al. 2001).

In contrast, analysis of the basic helix-loop-helix (bHLH) transcription factor dHAND (or HAND2) in zebrafish (Yelon et al. 2000), chicken (Fernandez-Teran et al. 2000), and mouse embryos (Srivastava et al. 1997; Charité et al. 2000) shows that dHAND is required for establishment of SHH signaling in both fin and limb buds. During embryonic development, $d H A N D$ is initially expressed throughout the flank mesenchyme and becomes restricted to the posterior mesenchyme during initiation of limb bud development (for review, see Cohn 2000). Therefore, identification of the gene(s) restricting dHAND to the posterior limb bud mesenchyme should provide insights into the genetic networks acting upstream of the polarizing region.

Disruption of the zinc finger protein GLI3 in the mouse results in establishment of a small anterior ectopic polarizing region and a polydactylous Extratoes $(X t)$ limb phenotype (Schimmang et al. 1992; Hui and Joyner 1993; Buscher et al. 1997). Normally, the full-length GLI3 zinc finger protein is processed to a transcriptional repressor (Wang et al. 2000), which participates in maintaining posterior restriction of $\mathrm{SHH}$ signaling during limb bud morphogenesis. SHH signaling by the polarizing region, in turn, inhibits the constitutive processing of GLI3, which results in formation of an anterior (high) to posterior (low) GLI3-R protein gradient (Wang et al. 2000). In addition to these reciprocal SHH-GLI3 interactions, genetic evidence for early GLI3 functions in establishing the polarizing region was also obtained (Zuniga and Zeller 1999). GLI3 is part of a larger number of genes whose disruption in the mouse results in establishment of an anterior SHH signaling center in limb buds and polydactyly (Masuya et al. 1995, 1997; Qu et al. 1997). Only a few of the disrupted genes have been identified, for example, the paired homeodomain transcription factor Aristaless-like4 $(A l \times 4)$. Disruption of the Alx4 gene in the mouse causes Strong's Luxoid (Lst) polydactyly, and it has been proposed that ALX4, like GLI3, is part of the genetic mechanism that keeps SHH signaling restricted to the posterior limb bud mesenchyme (Qu et al. 1997; Takahashi et al. 1998).

In the present study, we establish that GLI3 is required to restrict dHAND expression to the posterior mesen- 
chyme during initiation of limb bud morphogenesis. We also show that up-regulation of $A 1 \times 4$ expression depends on GLI3 function, thereby establishing that GLI3 acts initially upstream of ALX4. In turn, posterior dHAND function is required to keep both GLI3 and ALX4 restricted to anterior limb bud mesenchyme. Furthermore, dHAND positively regulates Gremlin expression and thereby differential responsiveness to $\mathrm{SHH}$ signaling, which, in turn, triggers establishment of the SHH/FGF feedback loop. Our studies reveal that the nascent limb bud mesenchyme is prepatterned by these genetic interactions prior to polarizing region signaling.

\section{Results}

\section{GLI3 restricts dHAND to the posterior limb bud mesenchyme during initiation of limb bud outgrowth}

dHAND is initially expressed throughout the lateral plate mesenchyme (Charité et al. 2000; Fernandez-Teran et al. 2000), but is restricted to the posterior mesenchyme during initiation of forelimb bud development by embryonic day 9.25 (E9.25; 22-23 somites; Fig. 1A). This correlates with activation of Gli3 expression in the anterior limb bud mesenchyme (Fig. 1B; see also Masuya et al. 1997; Zuniga and Zeller 1999). Therefore, the distribution of dHAND was determined in forelimb buds of $X t / X t$ homozygous embryos, which lack GLI3 function (Schimmang et al. 1992; Hui and Joyner 1993). In con-

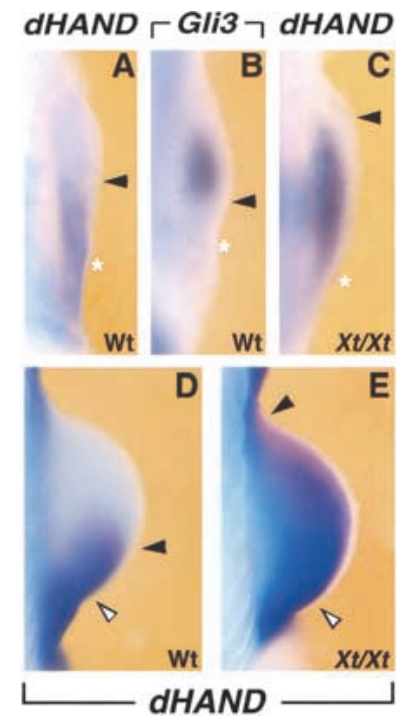

Figure 1. $d H A N D$ expression is restricted to the posterior limb bud mesenchyme by Gli3 repressor activity. (A) dHAND expression in the limb bud of a wild-type embryo (22-23 somites). dHAND expression is restricted to the posterior limb bud mesenchyme. $(B)$ Gli3 expression in the limb bud mesenchyme of a wild-type embryo (22-23 somites). (C) dHAND expression extends anteriorly in the limb bud of an $X t / X t$ homozygous embryo (22-23 somites). $(D, E)$ Expression of $d H A N D$ in limb buds of wild-type $(D)$ and $X t / X t(E)$ mutant embryos with 27 somites. $d H A N D$ expression is up-regulated in the posterior mesenchyme under the influence of SHH signaling (open arrowheads). All limb buds shown are forelimb buds. Arrowheads indicate boundaries of expression domains; asterisks in $A-C$ indicate the posterior margins of forelimb buds, with anterior to the top and posterior to the bottom. trast to the rapid posterior restriction in wild-type limb buds (Fig. 1A, arrowheads), dHAND expression persists in anterior mesenchyme of Gli3-deficient limb buds (2223 somites; Fig. 1C). During progression of limb bud development, $d H A N D$ remains expressed in the anterior limb bud mesenchyme of $X t / X t$ embryos $(27$ somites; Fig. 1, cf. E and D). In Gli3-deficient limb buds, $d H A N D$ expression is lower in anterior than in posterior mesenchyme (Fig. 1E, open arrowhead) as $d H A N D$ expression is up-regulated under the influence of $\mathrm{SHH}$ signaling (Charité et al. 2000; Fernandez-Teran et al. 2000). Shh expression is first detected in posterior forelimb bud mesenchyme around E9.5 (25 somites; Masuya et al. 1997; data not shown). The observed polarized expression of Gli3 (25 somites; Fig. 2A) and dHAND (Fig. 2B) is initially SHH-independent, as their complementary distribution is maintained in Shh-deficient limb buds (25 somites; Fig. 2C,D). During subsequent limb bud development, $\mathrm{SHH}$ signaling in posterior mesenchyme regulates Gli3 expression negatively (Marigo et al. 1996) and dHAND positively (Charité et al. 2000; Fernandez-Teran et al. 2000). In older Shh-deficient limb buds, GLI3 is no longer excluded from posterior-most mesenchyme (Fig. 2E; see also Chiang et al. 2001), whereas the dHAND domain fails to extend distally (cf. Fig. $2 \mathrm{~F}$ to $1 \mathrm{D}$; see also Charité et al. 2000).

Expression of the transcription factor $A l \times 4$ is activated in anterior limb bud mesenchyme with kinetics similar to Gli3. Therefore, the possibility that ALX4 could participate in restricting $d H A N D$ was also studied (Fig. 2G$\mathrm{L})$. In the absence of ALX4 function in Lst/Lst mouse embryos (25 somites), both posterior restriction of dHAND (Fig. 2, cf. G and $\mathrm{H}$ ) and expression of Gli3 (data not shown) are normal. In contrast, comparative analysis of Alx4 in wild-type and $X t / X t$ mutant limb buds shows that Alx4 expression is initially lower in Gli3-deficient limb buds (25 somites; Fig. 2, cf. I and J). Subsequently, $A l \times 4$ expression remains relatively restricted in $X t / X t$ mutant limb buds (34 somites; Fig. 2, cf. K and L). The results presented in Figures 1 and 2 show that GLI3 represses $d H A N D$ and is necessary for positive regulation of $A l \times 4$ in anterior mesenchyme.

\section{dHAND keeps both GLI3 and ALX4 anteriorly restricted during onset of limb bud outgrowth}

Previous analysis of $d H A N D$ mutant mouse embryos has established that morphogenesis of their limb buds is disrupted at an early stage. $d H A N D$-deficient limb buds appear slightly smaller than their wild-type counterparts, and activation of both 5 'HoxD genes and Shh around E9.5 is disrupted (Charité et al. 2000; Yelon et al. 2000). To assess whether expression of anterior genes is also altered in $d H A N D$ mutant limb buds, the distributions of GLI3 and ALX4 were analyzed. Whereas Gli3 is normally not expressed by posterior mesenchymal cells (Fig. 3A,C, arrowhead; see also Fig. 1B), it is expressed by posterior-most mesenchyme in $d H A N D$-deficient forelimb buds from early stages onward (Fig. 3B,D, arrowheads). Similar to Gli3, the expression of $A l \times 4$ is no longer restricted to the anterior-most mesenchyme (Fig. $3 \mathrm{E})$, but is expanded posteriorly in $d H A N D$-mutant limb buds (Fig. 3F). Taken together, these results show that both Gli3 and $A l \times 4$ are aberrantly expressed in posterior mesenchyme of $d H A N D$ mutant limb buds. 
However, one possible explanation for the observed nonpolarized Gli3 and $A l \times 4$ expression could be the loss of posterior mesenchyme. Hoxb8 is the earliest known marker for the posterior part of the forelimb field (Charité et al. 1994). By E9.5 (24-25 somites), weak Hoxb8 expression is still observed in the posterior half of both wild-type and $d H A N D$ mutant forelimb buds (Fig. 3G,H), which shows that posterior mesenchyme is not lost in dHAND mutant limb buds at this stage. As dHAND is essential for survival of mesenchymal cells (Srivastava et al. 1997), TUNEL staining was used to assess a possible contribution of apoptosis to these early alterations of gene expression patterns. Analysis of serial sections revealed that no significant increase in cell death has occurred in the mesenchyme of both wild-type and $d H A N D$ mutant forelimb buds by E9.5 (24-25 somites; Fig. 3I,J), when expression of Gli3 and $A l \times 4$ has already been expanded posteriorly (Fig. 3B,D,F). However, the mesenchyme of $d H A N D$ mutant limb buds begins to undergo massive apoptosis by E9.75 (27 somites and older; data not shown). In summary, the results shown in Figure 3 establish that dHAND function is required to exclude Gli3 and Alx4 from the posterior limb bud mesenchyme.

The reciprocal GLI3-dHAND interactions participate in establishing differential responsiveness to future SHH signaling

The BMP antagonist GREMLIN participates in relaying the $\mathrm{SHH}$ signal from the mesenchyme to the AER during establishment and maintenance of the SHH/FGF feedback loop. Activation of Gremlin in the limb bud mesenchyme and Fgf expression in the posterior AER precedes SHH signaling (Zuniga et al. 1999). Interestingly, the expression of both Gremlin (Fig. 4B,D) and Fgf4 (Fig. 4F) is anteriorly expanded in $X t / X t$ limb buds (Fig. 4, cf. A,C,E to B,D,F). This anterior expansion has occurred by E9.75 (29-30 somites; Fig. 4B) and precedes detection of ectopic anterior SHH signaling in $X t / X t$ limb buds by $\sim 1$ embryonic day (Buscher et al. 1997; Zuniga and Zeller 1999). In contrast, Gremlin expression is normal in Alx4-deficient limb buds during the same developmental period (data not shown).

One possible cause for this anterior expansion of Gremlin could be its up-

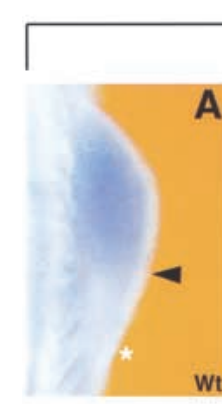
to the bottom.

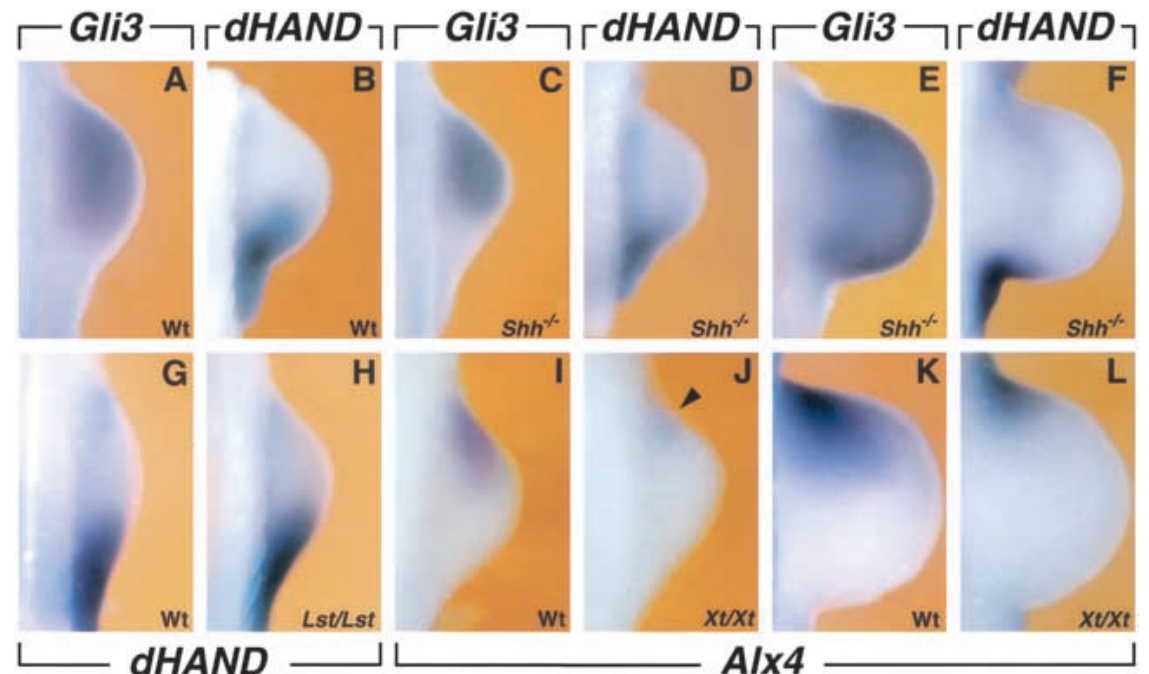

Figure 2. Complementary expression of Gli3 and $d H A N D$ is independent of SHH signaling and ALX4 function. $(A, B)$ Complementary expression of Gli3 $(A)$ and $d H A N D(B)$ in contralateral limb buds of a wild-type embryo (25 somites). (C,D) Complementary expression of Gli3 $(C)$ and $d H A N D(D)$ in contralateral limb buds of an Shh-deficient embryo (25 somites). $(E, F)$ Expression of Gli3 $(E)$ and $d H A N D(F)$ in contralateral limb buds of older Shh-deficient embryos (34 somites). $(G, H)$ dHAND expression in wild-type $(G)$ and Lst/Lst $(H)$ mutant limb buds (25 somites). (I-L) Comparative analysis of $A I_{X} 4$ expression in wild-type and $X t / X t$ homozygous limb buds. (I) Wild-type (25 somites); (J) Xt/Xt homozygous (25 somites), arrowhead points to weak Alx4 expression; $(K)$ wild-type (34 somites); $(L) X t / X t$ homozygous (34 somites). All limb buds shown are forelimb buds, with anterior to the top and posterior to the bottom. ever, dHAND is not necessary for Gremlin activation, as

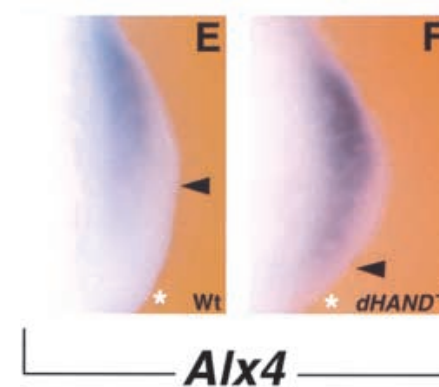
Gli3

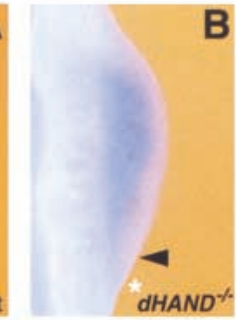
B
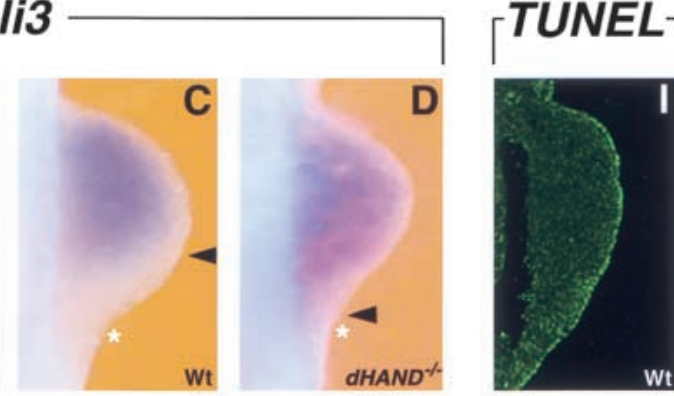

Figure 3. dHAND keeps Gli3 and Alx4 from being expressed by posterior limb bud mesenchyme. $(A-D)$ Gli3 expression in limb buds of wild-type $(A, C)$ and $d H A N D$-deficient $(B, D)$ embryos ( $A, B: 22$ somites; $C, D: 24-25$ somites). (E,F) Alx4 expression in limb buds of wildtype $(E)$ and $d H A N D$-deficient $(F)$ embryos (24 somites). $(G, H)$ Expression of Hoxb8 in limb buds contralateral to the ones shown in panels $C$ and $D$. $(G)$ Wild-type limb bud (24-25 somites); $(H)$ dHAND mutant limb bud (24-25 somites). Arrowheads in panels $A-H$ indicate posterior boundaries of expression domains. Asterisks indicate posterior edges of limb buds. $(I, J)$ TUNEL analysis to detect apoptotic cells. (I) Wild-type limb bud ( 24 somites); $(J) d H A N D$ mutant limb bud (24 somites). White arrowhead points to apoptotic cells in a somite (Srivastava et al. 1997). All limb buds shown are forelimb buds, with anterior to the top and posterior 


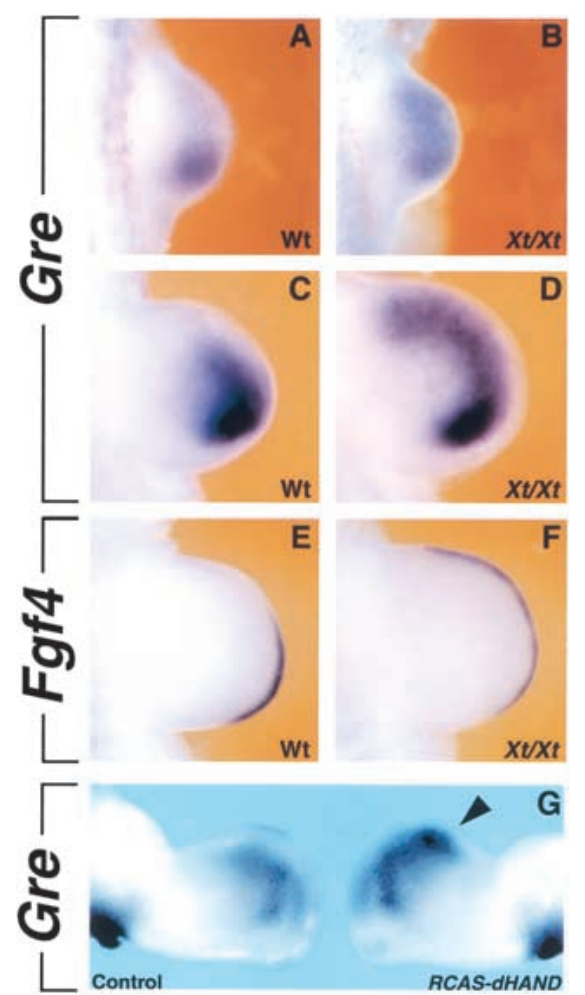

Figure 4. Genetic interaction of GLI3 and dHAND restricts GREMLIN-mediated competence to establish the SHH/FGF signaling feedback loop to the posterior limb bud mesenchyme. (A) Gremlin expression in a wild-type limb bud (29-30 somites). (B) Gremlin expression expands anteriorly in an Xt/Xt limb bud (29-30 somites). (C) Gremlin expression in a wild-type limb bud (37 somites). (D) Gremlin expression in an Xt/Xt limb bud (37 somites). (E,F) Fgf4 expression in the limb buds contralateral to the ones shown in panels $C$ and $D$. (E) Wild-type limb bud (37 somites); (F) Xt/Xt limb bud (37 somites). (G) Retroviral overexpression of $d H A N D$ in chicken limb buds results in similar up-regulation of Gremlin expression in the anterior mesenchyme (arrowhead) in all embryos analyzed $(n=6)$. All limb buds shown are forelimb buds, with anterior to the top and posterior to the bottom.

its expression is normal in $d H A N D$-deficient limb buds around E9.5 (Charité et al. 2000; data not shown). To determine if ectopic dHAND can up-regulate Gremlin in anterior mesenchyme, the prospective wing bud region of chicken embryos was infected with a retrovirus encoding the dHAND protein. Such ectopic dHAND expression induces weak anterior SHH signaling and results in duplication of anterior digits in a fraction of all wing buds (for details, see Fernandez-Teran et al. 2000). In contrast, dHAND overexpression causes anterior upregulation of Gremlin (Fig. 4G, arrowhead, embryonic stage 25$)$ in all cases $(n=6)$. The Gremlin domain in such wing buds is similar to what is observed in Gli3-deficient limb buds (Fig. 4, cf. G and D).

\section{Discussion}

As summarized in Figure 5, the present study uncovers components of a regulatory mechanism that prepatterns the limb bud mesenchyme prior to SHH signaling by the polarizing region. $d H A N D$ is initially expressed by the lateral plate mesenchyme and becomes restricted to the posterior mesenchyme during initiation of limb bud morphogenesis (Charité et al. 2000; Fernandez-Teran et al. 2000). Interestingly, this dynamic dHAND distribution largely parallels tissue competence to establish a polarizing region and activate SHH signaling. This competence is rather widespread but weak in flank mesenchyme prior to formation of limb buds (Tanaka et al. 2000). During initiation of limb bud outgrowth, both dHAND and the competence become restricted to and up-regulated in posterior mesenchyme. Indeed, genetic analysis of mouse and zebrafish embryos shows that dHAND is required to establish SHH signaling by the polarizing region in tetrapod limb buds (for review, see Cohn 2000). We now establish that GLI3-mediated transcriptional repression is crucial for restricting $d H A N D$ expression to the posterior mesenchyme (Fig. 5, pathway 1) concurrent with restriction of the competence to activate SHH signaling (Tanaka et al. 2000). Despite phenotypic and molecular similarities in the polydactylous limb phenotypes of Gli3- and Alx4-deficient mouse embryos (Qu et al. 1997; Takahashi et al. 1998), the posterior restriction of dHAND does not depend on ALX4 function. Rather, GLI3 function is required for positive regulation of Alx4 expression, which places GLI3 genetically upstream of Alx4 during initiation of limb bud morphogenesis (Fig. 5, pathway 2).

dHAND is genetically required to keep both Gli3 and Alx4 expression restricted to the anterior mesenchyme (Fig. 5, pathway 3). However, ectopic $d H A N D$ expression in chicken limb buds does not suffice to significantly down-regulate Gli3 and/or Alx4 in anterior mesenchyme (Fernandez-Teran et al. 2000). The repression of Gli3 and Alx4 may simply depend on formation of an active heterodimer between dHAND and another bHLH transcription factor (Firulli et al. 2000) expressed only in posterior mesenchyme. In addition, dHAND is required for transcriptional activation of several types of posterior patterning genes (Fig. 5, pathway 4), such as $5^{\prime} H o x D$ genes, Shh, and Bmp2 (Yelon et al. 2000). Interestingly, dHAND also regulates Gremlin positively, which, in turn, is part of the genetic cascades positioning the polarizing region and maintaining the SHH/FGF feedback

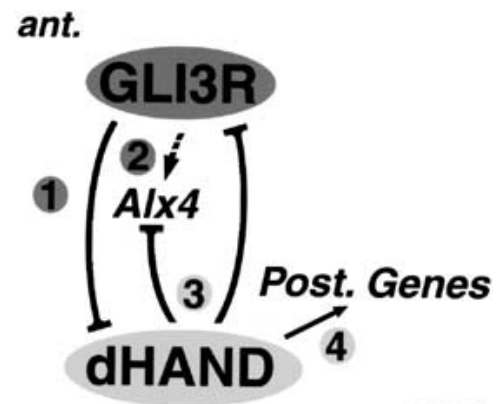

post.

Figure 5. Reciprocal genetic repression between GLI3 and dHAND prepatterns the limb bud mesenchyme prior to activation of $\mathrm{SHH}$ signaling. (1) GLI3 repressor activity (GLI3-R) restricts expression of the bHLH transcription factor $d H A N D$ to the posterior mesenchyme during onset of limb bud morphogenesis. (2) GLI3-R participates in positive transcriptional regulation (dashed arrow) of another anterior transcription factor, Alx4. (3) dHAND is necessary to keep Gli3 and Alx4 expression restricted to the anterior mesenchyme. (4) In posterior mesenchyme, dHAND is necessary for activating expression of posterior genes, among them $5^{\prime} H o x D$ genes, Bmp2, and Shh (for details and references, see text). These genetic interactions prepattern the limb bud mesenchyme independent of SHH signaling. 
loop (Zuniga and Zeller 1999; Zuniga et al. 1999). Therefore, loss of posterior restriction of dHAND in Gli3-deficient limb buds is a likely cause of the anterior expansion of the 5'HoxD (Zuniga and Zeller 1999) and Gremlin expression domains. This expansion long precedes establishment of a small anterior $\mathrm{SHH}$ signaling center. The analysis of Shh-deficient limb buds led Chiang et al. (2001) to conclude that the nascent limb field and early limb bud mesenchyme are prepatterned by an SHH-independent mechanism. The present study begins to uncover the molecular basis of this prepatterning mechanism and establishes that active cross-regulation between anterior and posterior mesenchyme is essential during initiation of limb bud outgrowth (Fig. 5). This prepatterning mechanism participates in determining posterior identity and positioning of the polarizing region and sets up differential mesenchymal responsiveness to future SHH signaling. As GLI3 functions first to restrict $d H A N D$ expression to posterior mesenchyme, establishment of the limb bud organizer seems triggered by anterior to posterior repression of activators rather than solely by posterior activation.

\section{Materials and methods}

Mouse strains and embryos

Gli3-deficient mouse embryos were obtained by intercrossing heterozygous mice carrying the $X t^{J}$ allele. The $3^{\prime}$ part of the Gli3 gene is deleted in the $X t^{I}$ allele, and mutant embryos were PCR-genotyped as described by Buscher et al. (1997). Alx4-deficient mouse embryos were obtained by intercrossing heterozygous mice carrying the $L s t^{I}$ allele. $L s t^{I}$ embryos were PCR-genotyped using a strategy based on the 16-bp deletion within the homeobox domain of the $A l_{x} 4$ gene (Takahashi et al. 1998). $d H A N D$ deficient embryos were obtained by intercrossing $d H A N D$ heterozygous mice and genotyped as described by Srivastava et al. (1997). Shh-deficient embryos were obtained by intercrossing heterozygous Shh mice and genotyped as described by St-Jacques et al. (1998).

Whole-mount in situ hybridization

Whole-mount in situ hybridization using digoxygenin-labeled RNA probes was performed as described by Haramis et al. (1995). The chicken Gremlin probe was isolated by RT-PCR and its identity confirmed by DNA sequencing. Wild-type and mutant embryos were age-matched according to their somite numbers (variation \pm 2 somites). Reproducibility of all results was ensured by analyzing several embryos $(n \geq 3)$ in independent experiments.

Retroviral infection of chicken wing buds

RCAS- $d H A N D$ retroviral particles were injected into the presumptive wing field of chicken embryos (stage 12-14), and embryos were analyzed by in situ hybridization 3 d later (stage 25), as described in detail by Fernandez-Teran et al. (2000). Pathogen-free eggs (CRIFFA) were used for all studies, and embryos were staged according to Hamburger and Hamilton (1951).

\section{Detection of apoptotic cells by TUNEL staining}

Apoptotic cells were detected in situ by incorporating fluorescein-dUTP into fragmented DNA using terminal transferase according to the manufacturer's instructions (Roche Diagnostics).

\section{Acknowledgments}

We thank A. Beverdam $\left(L s t^{\top}\right)$ and D. Srivastava (dHAND) for making colony founders and probes for genotyping available to us. R. Dono, F. Meijlink, and A. Zuniga are thanked for reagents, advice, and many helpful discussions. We are grateful to S. Cohen, J. Deschamps, T. Durston, B. Scheres, and members of our groups for comments on the manuscript. This study is supported by NWO Grant 810.68 .012 (R.Z.); the Faculty of Biology, Utrecht University (R.Z); and the Spanish Ministry of Science Grants DGICYT-PM98-0151 and FIS 01/1219 (M.R).
The publication costs of this article were defrayed in part by payment of page charges. This article must therefore be hereby marked "advertisement" in accordance with 18 USC section 1734 solely to indicate this fact.

\section{References}

Buscher, D., Bosse, B., Heymer, J., and Ruther, U. 1997. Evidence for genetic control of Sonic hedgehog by Gli3 in mouse limb development. Mech. Dev. 1997: 175-182.

Charité, J., de Graaff, W., Shen, S., and Deschamps, J. 1994. Ectopic expression of Hoxb- 8 causes duplication of the ZPA in the forelimb and homeotic transformation of axial structures. Cell 78: 589-601.

Charité, J., McFadden, D.G., and Olson, E.N. 2000. The bHLH transcription factor dHAND controls Sonic hedgehog expression and establishment of the zone of polarizing activity during limb development. Development 127: 2461-2470.

Chiang, C., Litingtung, Y., Harris, M.P., Simandl, B.K., Li, Y., Beachy, P.A., and Fallon, J.F. 2001. Manifestation of the limb prepattern: Limb development in the absence of Sonic hedgehog function. Dev. Biol. 236: 421-435.

Cohn, M.J. 2000. Giving limbs a hand. Nature 406: 953-954.

Fernandez-Teran, M., Piedra, M.E., Kathiriya, I.S., Srivastava, D., Rodriguez-Rey, J.C., and Ros, M.A. 2000. Role of dHAND in the anteriorposterior polarization of the limb bud: Implications for the Sonic hedgehog pathway. Development 127: 2133-2142.

Firulli, B.A., Hadzic, D.B., McDaid, J.R., and Firulli, A.B. 2000. The basic helix-loop-helix transcription factors $d H A N D$ and $e H A N D$ exhibit dimerization characteristics that suggest complex regulation of function. J. Biol. Chem. 275: 33567-33573.

Hamburger, V. and Hamilton, H. 1951. A series of normal stages in the development of the chick embryo. J. Morphol. 88: 49-92.

Haramis, A.G., Brown, J.M., and Zeller, R. 1995. The limb deformity mutation disrupts the SHH/FGF-4 feedback loop and regulation of 5'HoxD genes during limb pattern formation. Development 121: 4237-4245.

Hui, C. and Joyner, A. 1993. A mouse model of greig cephalopolysyndactyly syndrome: The extra-toes mutation contains an intragenic deletion of the Gli3 gene. Nat. Genet. 3: 241-246.

Johnson, R.L. and Tabin, C.J. 1997. Molecular models for vertebrate limb development. Cell 90: 979-990.

Kraus, P., Fraidenraich, D., and Loomis, C.A. 2001. Some distal limb structures develop in mice lacking Sonic hedgehog signaling. Mech. Dev. 100: 45-58.

Lu, H.C., Revelli, J.P., Goering, L., Thaller, C., and Eichele, G. 1997. Retinoid signalling is required for the establishment of a ZPA and for the expression of Hoxb-8, a mediator of ZPA formation. Development 124: 1643-1651.

Marigo, V., Johnson, R.L., Vortkamp, A., and Tabin, C.J. 1996. Sonic hedgehog differentially regulates expression of Gli and Gli3 during limb development. Dev. Biol. 180: 273-283.

Masuya, H., Tomoko, S., Wakana, S., Moriwaki, K., and Shiroishi, T. 1995. A duplicated zone of polarizing activity in polydactylous mouse mutants. Genes \& Dev. 9: 1645-1653.

Masuya, H., Sagai, T., Moriwaki, K., and Shiroishi, T. 1997. Multigenic control of the localization of the zone of polarizing activity in limb morphogenesis in the mouse. Dev. Biol. 182: 42-51.

Qu, S., Niswender, K., Ji, Q., van der Meer, R., Keeny, D., Magnuson, M.A., and Wisdom, R. 1997. Polydactyly and ectopic ZPA formation in AlX-4 mutant mice. Development 124: 3999-4008.

Schimmang, T., Lemaistre, M., Vortkamp, A., and Rüther, U. 1992. Expression of the zinc finger gene Gli3 is affected in the morphogenetic mouse mutant extra-toes (Xt). Development 116: 799-804.

Srivastava, D., Thomas, T., Lin, Q., Kirby, M.L., Brown, D., and Olson, E.N. 1997. Regulation of cardiac mesodermal and neural crest development by the bHLH transcription factor dHAND. Nat. Genet. 16: $154-160$

St-Jacques, B., Dassule, H., Karavanova, I., Botchkarev, V., Li, J., Danielian, P., McMahon, J., Lewis, P., Paus, R., and McMahon, A. 1998. Sonic hedgehog signaling is essential for hair development. Curr. Biol. 8: 1058-1068.

Sun, X., Lewandoski, M., Meyers, E.N., Liu, Y.H., Maxson, R.E., Jr., and Martin, G.R. 2000. Conditional inactivation of Fgf4 reveals complex- 


\section{te Welscher et al.}

ity of signalling during limb bud development. Nat. Genet. 25: 83-86.

Takahashi, M., Tamura, K., Buescher, D., Masuya, H., Yonei-Tamura, S., Matsumoto, K., Naitoh-Matsuo, M., Takeuchi, J., Ogura, K., Shiroishi, T., et al. 1998. The role of Alx-4 in the establishment of anteroposterior polarity during vertebrate limb development. Development 125: 4417-4425.

Tanaka, M., Cohn, M.J., Ashby, P., Davey, M., Martin, P., and Tickle, C. 2000. Distribution of polarizing activity and potential for limb formation in mouse and chick embryos and possible relationships to polydactyly. Development 127: 4011-4021.

van den Akker, E., Reijnen, M., Korving, J., Brouwer, A., Meijlink, F., and Deschamps, J. 1999. Targeted inactivation of Hoxb8 affects survival of a spinal ganglion and causes aberrant limb reflexes. Mech. Dev. 89: $103-114$

van den Akker, E., Fromental-Ramain, C., de Graaff, W., Le Mouellic, H., Brulet, P., Chambon, P., and Deschamps, J. 2001. Axial skeletal patterning in mice lacking all paralogous group 8 Hox genes. Development 128: 1911-1921.

Wang, B., Fallon, J.F., and Beachy, P.A. 2000. Hedgehog-regulated processing of Gli3 produces an anterior/posterior repressor gradient in the developing vertebrate limb. Cell 100: 423-434.

Yelon, D., Baruch, T., Halpern, M.E., Ruvinsky, I., Ho, R.K., Silver, L.M. and Stainier, D.Y.R. 2000. The bHLH transcription factor Hand2 plays parallel roles in zebrafish heart and pectoral fin development. Development 127: 2573-2582.

Zuniga, A. and Zeller, R. 1999. Gli3 (Xt) and formin (ld) participate in the positioning of the polarising region and control of posterior limb-bud identity. Development 126: 13-21.

Zuniga, A., Haramis, A.P., McMahon, A.P., and Zeller, R. 1999. Signal relay by BMP antagonism controls the SHH/FGF4 feedback loop in vertebrate limb buds. Nature 401: 598-602. 


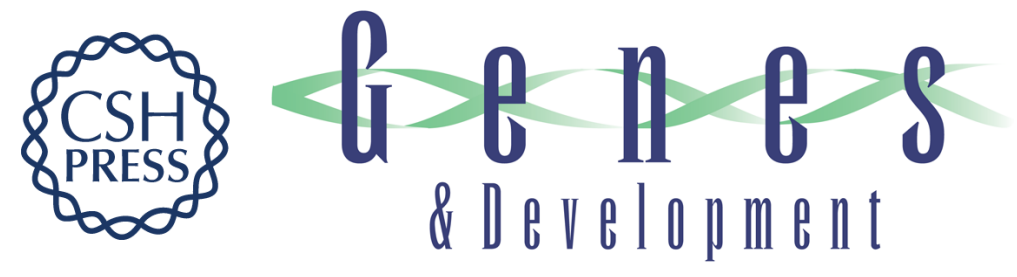

\section{Mutual genetic antagonism involving GLI3 and dHAND prepatterns the vertebrate limb bud mesenchyme prior to SHH signaling}

Pascal te Welscher, Marian Fernandez-Teran, Marian A. Ros, et al.

Genes Dev. 2002, 16:

Access the most recent version at doi:10.1101/gad.219202

$\begin{array}{ll}\text { References } & \begin{array}{l}\text { This article cites } 28 \text { articles, } 13 \text { of which can be accessed free at: } \\ \text { http://genesdev.cshlp.org/content/16/4/421.full.html\#ref-list-1 }\end{array}\end{array}$

License

Email Alerting

Receive free email alerts when new articles cite this article - sign up in the box at the top Service right corner of the article or click here.

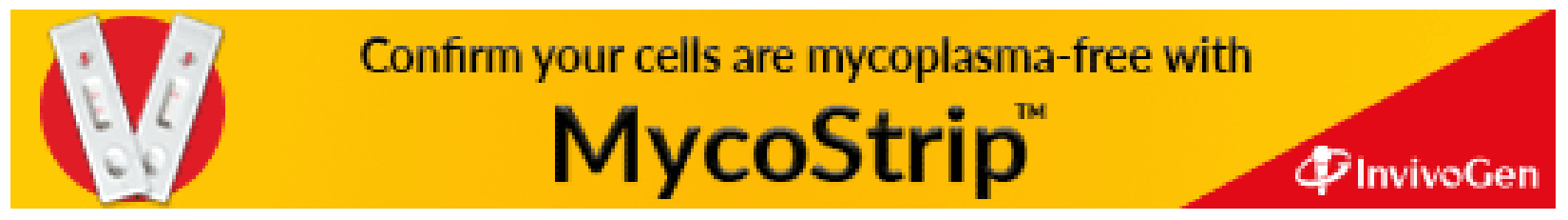

